\title{
Perisylvian functional connectivity during processing of sentential negation
}

\author{
Jörg Bahlmann ${ }^{1,2 *}$, Jutta L. Mueller ${ }^{1}$, Michiru Makuuchi' ${ }^{1}$ and Angela D. Friederici ${ }^{1}$ \\ Max Planck Institute for Human Cognitive and Brain Sciences, Leipzig, Germany \\ 2 Helen Wills Neuroscience Institute, University of California at Berkeley, Berkeley, CA, USA
}

\section{Edited by:}

Marcela G. Pena, University of Chile,

Chile

Reviewed by:

Satoru Yokoyama, Tohoku University, Japan

Andrea Moro, Istituto Universitario di

Studi Superiori di Pavia, Italy

\section{${ }^{*}$ Correspondence:}

Jörg Bahlmann, Helen Wills

Neuroscience Institute, University of California at Berkeley, 132 Barker Hall,

Berkeley, CA 94720, USA.

e-mail: bahlmann@berkeley.edu
Every language has the means to reverse the truth value of a sentence by using specific linguistic markers of negation. In the present study we investigated the neural processing costs afforded by the construction of meaning in German sentences containing negation in different clause types. We studied negations within and across clause boundaries as well as single and double negations. Participants read German sentences comprising of affirmations, single negations in the main or in the subordinate clause, or double negations. As a result, we found a network including the left inferior frontal gyrus (pars triangularis, BA 45), and the left inferior parietal gyrus (BA 40) to be activated whenever negations in the main clause had to be processed. Additionally, we found increased functional coupling between the left pars triangularis (BA 45), left pars opercularis (BA 44), left SMA (BA 6), and left superior temporal gyrus (BA 42) during the processing of main clause negations. The study shows that in order to process negations that require semantic integration across clause boundaries left BA 45 interplays with other areas that have been related to language processing and/or the processing of cognitive demands and logical/conditional reasoning. Thus, the results indicate that the left perisylvian language network synchronizes in order to resolve negations, in particular, whenever requirements on meaning integration are enhanced.

Keywords: language, negation, semantic, fMRI, functional connectivity

\section{INTRODUCTION}

Language provides us with a very efficient means to reverse the truth value of a sentence or a sentence constituent. In English, we just need to add the morpheme /not/ in "Peter did not see Mary." versus "Peter did see Mary." in order to change an affirmative proposition into a negative one. The linguistic operation that flips the polarity of a sentence is termed sentential negation.

From behavioral studies there is much empirical support for the occurrence of processing difficulties during the comprehension of negated sentences. Just and Carpenter (1975) found negative sentences to be more difficult than affirmative sentences in a sentence-picture matching task. Kaup and Zwaan (2003) reported longer reaction times (RTs) for negative versus affirmative sentences in a probe recognition task. In a lexical decision task, Hasson and Glucksberg (2006) demonstrated that comprehension of negations is more difficult compared to comprehension of affirmations. As a whole, experiments investigating sentential negations showed increased processing costs for negative versus affirmative sentences.

In psycholinguistics these processing costs accompanying negation have been explained differently. Earlier approaches (e.g., Just and Carpenter, 1975) described the negation operator as an additional level in the propositional representation engendering additional processing time. More recent approaches favor explanations based on mental simulation processes of both the negated and the actual meaning of the sentence (e.g., Kaup and Zwaan, 2003; Hasson and Glucksberg, 2006). ERP research supports the notion of late and costly integration of negation. Lüdtke et al. (2008) investigated negations using a sentence-picture matching task and observed that negation was fully represented only at a relatively late time window (1500 ms after sentence offset) as indicated by an N400 response to a mismatch between the picture and the actual sentence meaning. Thus, behavioral as well as ERP research clearly indicates additional and costly processes during the processing of sentences including negation compared to their affirmative counterparts.

Interestingly, only a few imaging studies on sentential negations have been published so far. Carpenter et al. (1999) presented statements like "It is not true that the star is above the plus." Next, a picture was presented that corresponded to the sentence (a plus above a star), or not. Participants were asked to judge the correctness of the picture. As in the previous behavioral studies, RT was greater for the negative sentence condition. As for neural correlates, higher activity in the left posterior temporal and bilateral parietal brain regions was found for the negative compared to affirmative sentences describing spatial relations. In a study with Japanese participants, Hasegawa et al. (2002) used affirmative sentences like "The worker read a magazine and showed some pictures to the brother." versus sentences with a negation "The doctor read a book and didn't bring the baggage to the visitor." The sentences were followed by a true or false probe sentence. Sentences were presented in Japanese (first language, L1) and English (second language, L2). A significant effect for negative sentences was found in the left temporal and left precentral gyrus but only for the more demanding L2 condition. Christensen (2009) applied a similar probe-target matching task. Affirmative or negative target sentences like "The toughest men know but don't use soap." were followed by affirmative or negative 
probe sentences like "The toughest man use soap." Negative versus affirmative probe sentences generated increased activity in the left premotor cortex (BA 6). In another recent study Tettamanti et al. (2008) crossed the factor polarity (affirmative versus negative) with the factor concreteness (action-related versus abstract). Example: "Now I push the button" versus "Now I don't push the button." The main effect of concreteness (abstract > action-related) showed increased activations in the left hemispheric perisylvian and parietal areas. No increase of the hemodynamic response for the processing of negative sentences was observed. The only main effect of polarity (negative $>$ affirmative) that was found was a deactivation in the left pallidum, and the right middle frontal and middle occipital cortices. Additionally, the authors conducted a dynamic causal modeling analysis within the left hemispheric network (including inferior frontal and precentral gyrus, superior and inferior temporal gyrus, and supramarginal gyrus), which showed a sensitivity with respect to action-related sentences. They demonstrated, that not only the mere activation of single areas, but also the coupling between the areas in this network was reduced during the processing of negative sentences. The described fMRI studies investigated into syntactic (Tettamanti et al., 2008) or pragmatic (Carpenter et al., 1999; Hasegawa et al., 2002; Christensen, 2009) aspects of negation. However, the semantic aspect of negation remains to be investigated.

Overall, the three fMRI experiments on sentential negation deliver a rather heterogeneous activation pattern. The only consistency in the findings is that none of these studies found activity in the classical perisylvian language network including the left inferior frontal and the superior temporal cortex, when negative versus affirmative sentences were directly compared. This is surprising because left inferior frontal and superior temporal activation has been frequently reported for the processing of sentences that are more demanding either in the semantic or in the syntactic domain (e.g., Fiebach et al., 2005; Rodd et al., 2009). Even in the more demanding second language processing situation, which usually activates the inferior frontal cortex (e.g., Wartenburger et al., 2003), the left inferior frontal gyrus (IFG) was not engaged (Hasegawa et al., 2002). Behavioral experiments clearly suggest processing difficulties for negative versus affirmative sentences (e.g., Kaup and Zwaan, 2003). Nevertheless, the perisylvian language network appears to cope with this difficulty very well, since the discussed fMRI method did not detect increased activation for negations. One possible explanation could be that the fact that language comprehension is an "over-learned" skill causes a ceiling effect with respect to brain activations. Sentence processing correlates with increased activity in the perisylvian language areas. Possibly, the processing difficulties for negative sentences that were engendered by previous studies were not serious enough in order to add to this general hemodynamic and thus did not generate a distinguishable characteristic brain response for negation processing in the classic areas. Thus, the perisylvian language network is either not involved in the analysis of sentential negations, or the tasks that were used up-to-date in order to detect such involvement were not sensitive enough.

In the present study we aimed to investigate the processing of negations during online sentence comprehension in a graded manner in which we systematically varied the scope of negation processing. To achieve this we used four different sentence types. All sentences comprised of a main clause and a subordinate clause. The assertions in the clauses were either affirmative or negative. In our study, a negation in the main clause reverses the truth value of the subordinate clause while the scope of a negation in the subordinate clause is limited to the assertion within the clause. Negations were expressed by adding the adverbial marker "nicht". Sentences either comprised of (1) a negative assertion in the main and in the subordinate clause (NN), (2) an affirmative assertion in the main and a negative assertion in the subordinate clause (AN), (3) a negative assertion in the main and an affirmative assertion in the subordinate clause (NA), or (4) a affirmative assertion in the main and in the subordinate clause (AA). These conditions resulted in a $2 \times 2$ design with the factors POLARITY_MAIN and POLARITY_SUB. POLARITY_MAIN refers to negation or affirmation in the main clause of the sentences ( $N N$ and $A N$ ). POLARITY_SUB refers to negation or affirmation in the subordinate clauses of the sentences (NA and AA). Note that, a double negation represents a special case. In languages like Russian, French, or Italian the double negation describes an endorsement of a negation (e.g., Russian: "Ja ne snaju nitschewo" [I don't know nothing]). In contrast, in German and in English the parsing of a double negation is determined by the logical form, and is resolved as an affirmation (Partee et al., 1993; de Swart, 1998). For example in German, double negations are used relatively frequently in a discourse in relative clauses, e.g., A: "Du hast nichts gelernt" [You didn't learn anything.]; B: "Es ist nicht wahr, dass ich nichts gelernt habe." [It is not true, that I didn't learn anything.]. In the present study, we applied double negations like: "Es ist nicht wahr, dass Peter Hans nicht besuchte." [It is not true, that Peter did not visit Hans]. Such sentences are resolved as an affirmation. In such cases, the main clause "Es ist nicht wahr" flips the polarity of the subordinate clause "dass Peter Hans nicht besuchte." Thus, a double negation results in an affirmative interpretation of the sentence. Negation is considered to be related to the logical form of a sentence (e.g., Partee et al., 1993; de Swart, 1998). Hence, the processing of negation in the present study should reflect semantic operations during online sentence comprehension. Semantic operations include the incremental integration of word meanings into the meaning of phrases and sentences. Thus, semantic processing of the negative marker "nicht" is expected to lead to additional costs with respect to "semantic integration." The processing of semantics correlates with activity in the anterior portion of Broca's area. In particular the left pars triangularis (BA 45) and the left pars orbitalis (BA 47) seem to play a crucial role in the processing of semantics during sentence comprehension (e.g., Bookheimer, 2002). Behavioral and ERP studies suggest that there are higher integration demands of negative sentences compared to affirmative sentences (Kaup and Zwaan, 2003; Lüdtke et al., 2008). Thus, in the present study, we predict that the processing of negations in comparison to affirmations should lead to higher demands on semantic integration. These higher demands on semantic integration should reveal an engagement of the left BA 45/47 during the processing of negations. Further, as previous studies have shown that language processing always invokes the concurrent activation of a network of areas rather than local activation (Lohmann et al., 2010) we were particularly interested in the investigation of 
functional couplings between language-related areas during the processing of negations. To do so, we applied a very short repetition time $(\mathrm{TR}=1.5 \mathrm{~s})$ in order to maximize the measured time bins of the hemodynamic changes. We applied a psychophysiological interaction (PPI) analysis to examine the interplay of different brain regions during online processing of negated sentences.

\section{MATERIALS AND METHODS PARTICIPANTS}

Seventeen right-handed participants took part in the fMRI study (eight female, mean age $=24.5$ years, $\mathrm{SD}=2.8$ years). They were all native speakers of German and had normal, or corrected to normal vision. None of the participants had a history of neurological, major medical, or psychiatric disorder.

\section{STIMULI}

The experiment comprised of four different sentence types. All sentences had the same structure, namely a main clause followed by a subordinate clause (see Table 1 and Figure 1). The main clause either contained a negation "Es ist nicht wahr,..." [It is not true,...], or contained an affirmation "Es ist schon wahr,..." [It is indeed true,...]. Similarly, the subordinate clause comprised of a negation “... dass der Peter den Hans heute Morgen in der Pause nicht besuchte." [... that Peter did not visit Hans this morning during the break.]. Accordingly, an affirmation in the subordinate clause looked like this: “... dass der Peter den Hans heute Morgen in der Pause wirklich besuchte." [... that Peter really visited Hans this morning during the break.]. The different sentence types resulted in four different conditions. A double negation (NN) comprised of a negation in the main clause, as well as a negation in the subordinate clause. A single main clause negation (NA) had the form of a negation at the main clause and an affirmation in the subordinate clause. A single negation in the subordinate clause (AN) contained an affirmation in the main clause and a negation in the subordinate clause. Finally, the affirmative sentence (AA) has an affirmative content in both, the main and the subordinate clause. In the affirmative sentences the filler words "schon" [indeed] and "wirklich" [really] were included. This ensured that all sentences had the same amount of words. Moreover, the filler words occurred at the same position in a sentence, as the negation statement ("nicht" [not]).

Table 1: Experimental conditions.

\begin{tabular}{|c|c|}
\hline NN & AN \\
\hline Es ist nicht wahr, dass Peter & Es ist schon wahr, dass Peter Thomas \\
\hline Thomas letzte Woche für das & letzte Woche für das Projekt nicht \\
\hline Projekt nicht einstellte. & einstellte. \\
\hline It is not true, that Peter did not hire & It is indeed true, that Peter did not hire \\
\hline Thomas last week for the project. & Thomas last week for the project. \\
\hline NA & AA \\
\hline Es ist nicht wahr, dass Peter & Es ist schon wahr, dass Peter Thomas \\
\hline Thomas letzte Woche für das & letzte Woche für das Projekt wirklich \\
\hline Projekt wirklich einstellte. & einstellte. \\
\hline It is not true, that Peter really hired & It is indeed true, that Peter really hired \\
\hline Thomas last week for the project. & Thomas last week for the project. \\
\hline
\end{tabular}

The four different sentential conditions were tested in a $2 \times 2$ design, with the factors POLARITY_MAIN (negation versus affirmation in the main clause) and POLARITY_SUB (negation versus affirmation in the subordinate clause).

For each condition 32 different sentences were generated. The selection of the sentences proceeded as follows. Each subordinate clause started with two names (e.g., Peter Hans), followed by information about when (e.g., "heute Morgen" [this morning]), and where (e.g., "in der Pause" [during the break]), and ended with the verb (three syllables, regular inflection). The frequency was balanced according to the German lexicon using the CELEX database. In order to control, as far as possible, for lexical content across the different sentence types, we used the identical basis sentences in each of the four conditions. A total of 64 sentences per condition were generated. These sentences were rated in a behavioral preexperiment according to acceptability and plausibility. The resulting 32 sentences with the highest rating across conditions entered the fMRI experiment. For each target sentence a comprehension sentence was generated. The comprehension sentence was derived from the target sentence and was a question aiming at the polarity, the person, the temporal information, or the locative information contained in the target sentence. This manipulation ensured that participants read and understood the whole sentence, instead of just concentrating on the polarity of the sentence.

The experiment presentation was programmed with the Presentation 10.3 software (Neurobehavioral Systems, Inc.) on a Windows PC. Stimuli were projected through an LCD projector (PCL-XP50L, SANYO) onto the back of a screen. Participants viewed the images on the screen above their heads through a mirror attached to the head-coil.

\section{EXPERIMENTAL PROCEDURE}

Sentences were presented visually phrase-by-phrase in randomized order. Each of the six phrases of a sentence were presented at the center of the screen for $800 \mathrm{~ms}$ (+200 ms blank). Then, after an ISI

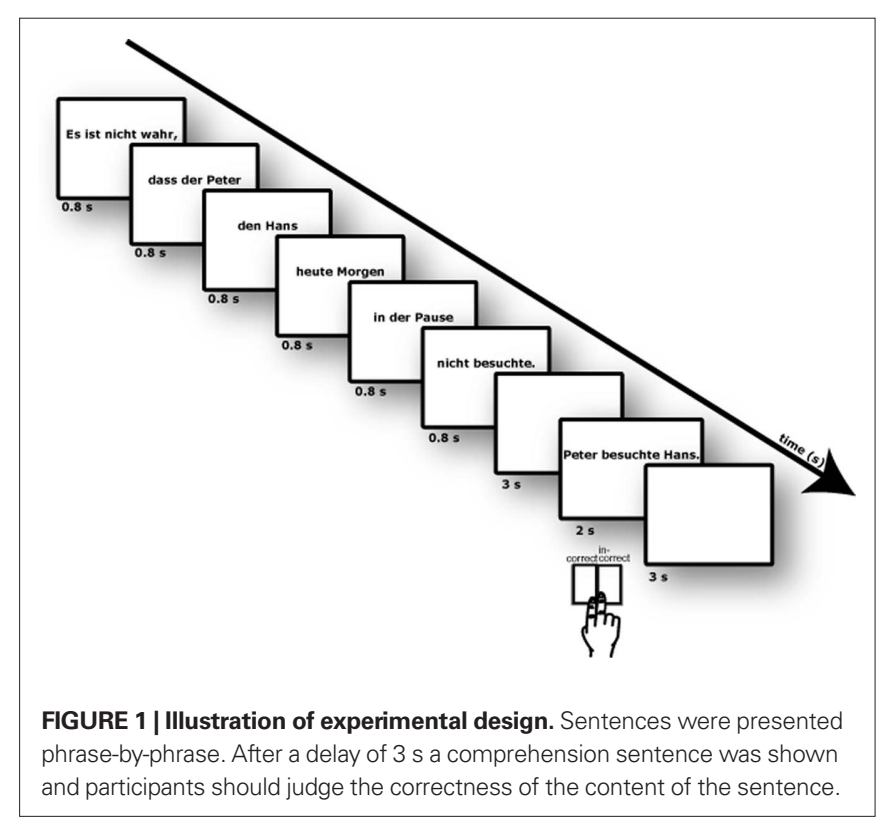


of $3000 \mathrm{~ms}$, a comprehension sentence was shown (2500 ms). This was followed by a short feedback ("correct"/ incorrect"; $500 \mathrm{~ms}$ ) and a blank screen with an ISI of $2000 \mathrm{~ms}$. Onsets of each trial were jittered against the scanning with 0, 500, 1000, and $1500 \mathrm{~ms}$. Mean sentence onset asynchrony was $15 \mathrm{~s}$. Thirty-two sentences per condition and 32 null-events (fixation cross, $15 \mathrm{~s}$ ) were given, resulting in a scanning time of $40 \mathrm{~min}$. During the presentation of the comprehension sentence participants were asked to provide a judgment regarding the content of the sentence. Participants were requested to judge whether the short sentence expressed the same content as the target sentence or not. They should deliver their judgment as soon as possible by pressing MRI-compatible response buttons, using the index and the middle finger of the right hand. Response-key assignment (right index finger versus right middle finger) was counterbalanced across participants.

\section{fMRI ACQUISITION}

Functional MRI data were acquired with a whole-body 3 T TRIO scanner (Siemens, Erlangen, Germany) with a gradient-echo echoplanar imaging (EPI) sequence. For registration purposes, two sets of two-dimensional anatomical images were acquired for each participant immediately prior to the functional imaging session. An MDEFT (data matrix $256 \times 256, \mathrm{TR}=1.3 \mathrm{~s}, \mathrm{TE}=7.4 \mathrm{~ms}$ ) and an EPI$\mathrm{T} 1$ ( $\mathrm{TE}=14 \mathrm{~ms}, \mathrm{TR}=3000 \mathrm{~ms})$ sequence were used. Additionally, geometric distortions were characterized by a B0 field-map scan. The field-map scan consisted of a gradient-echo readout (32 echoes, inter-echo time $0.64 \mathrm{~ms}$ ) with a standard two-dimensional phase encoding. The B0 field was obtained by a linear fit to the unwrapped phases of all odd echoes. Functional MRI scanning was carried out using a $\mathrm{T} 2{ }^{\star}$-weighted BOLD sensitive gradient-echo EPI sequence $\left(\mathrm{TR}=1.5 \mathrm{~s}, \mathrm{TE}=24 \mathrm{~ms}, \mathrm{FOV}=19.2 \mathrm{~cm}\right.$, flip angle $=65^{\circ}, 64 \times 64$ matrix, resulting in an in-plane resolution of $3 \mathrm{~mm} \times 3 \mathrm{~mm}$ ). Thirty slices (thickness: $3 \mathrm{~mm}$ with an interslice gap of $1 \mathrm{~mm}$ ) covering the whole brain were acquired. A parallel acquisition technique (Grappa, acceleration factor $=2$ ) was used in order to speed up the imaging readout (Griswold et al., 2002). Anatomical and functional images were positioned parallel to AC-PC. One functional run with 1605 volumes was collected. Stabilization cushions were used to reduce head motion.

\section{fMRI ANALYSIS}

MRI data were analyzed using SPM8 (available at http://www. fil.ion.ucl.ac.uk/spm) on a PC workstation. Pre-processing comprised of realignment and unwarp, slice timing, coregistration, segmentation, normalization to MNI space, and smoothing with a 8-mm full-width at half-maximum (FWHM) Gaussian kernel. Estimation of geometric distortion parameters for the realignment and unwarp procedure was conducted using the individual field maps. Normalizing an individual structural image to the SPM8 T1 brain template was processed in two steps: First, estimation of the normalization parameters. Second, writing the normalized images with the parameters. This parameter transformed the structural images and all EPI volumes into a common stereotactic space to allow for multisubject analyses. Voxel size was interpolated during pre-processing to isotropic $3 \mathrm{~mm} \times 3 \mathrm{~mm} \times 3 \mathrm{~mm}$. For the statistical analysis, the onset from each of the four conditions was modeled as an event of interest. Additionally, also the comprehension sentences and the baseline (fixation cross) were modeled as distinct conditions and were not mixed with the four conditions of interest. Confounds by global signal changes were removed by applying a high-pass filter with a cut-off frequency of $100 \mathrm{~s}$. In total, there were 32 events per condition. In the context of the general linear model, these events were convolved with a synthetic hemodynamic response function (with time and dispersion derivatives), yielding statistical parametric maps (Friston et al., 1995). Signal change relative to baseline in each condition was estimated. The resulting individual contrast images were submitted to the second-level analysis. A $2 \times 2$ ANOVA with the factors POLARITY_MAIN (negation versus affirmation in the main clause) and SUB (negation versus affirmation in the subordinated clause) as implemented in SPM8 was applied (including correction for non-sphericity). Main effects and interactions were calculated with linear contrasts ( $t$-test across the contrast images of all participants). To protect against false-positive activations a double threshold was applied, by which only regions with a $z$-score exceeding 3.09 ( $p<0.001$, uncorrected) and a volume exceeding $324 \mathrm{~mm}^{3}$ (corresponding to 12 resembled voxels) were considered (corresponding to $p<0.05$, corrected). This was determined in a Monte Carlo simulation using a Matlab script provided by Scott Slotnick (see http://www2.bc.edu/ slotnics/scripts.htm).

\section{VOLUME OF INTEREST ANALYSIS}

The data for the volume of interest (VOI) analysis was extracted from the brain regions found to be significantly activated in the main effect of POLARITY_MAIN in the whole-brain ANOVA. This analysis was conducted in order to further explore the influence of the four different conditions on the main effect. For each participant, an individual $6 \mathrm{~mm}$ radius spherical VOI was defined. The definition of the VOI was based on the peak coordinates of the random-effects analysis. Participants that showed no activity $(p<0.05)$ in a particular area were excluded from the analysis. For the analysis in the left IFG, left inferior parietal lobule (IPL), and right angular gyrus the data of one participant was excluded. The data of three participants were excluded from the analysis of the left precentral gyrus and right anterior insula. In all VOIs an ANOVA with the factors POLARITY_MAIN and POLARITY_SUB was conducted.

\section{PSYCHOPHYSIOLOGICAL INTERACTION ANALYSIS}

In order to explore which brain regions exhibit functional coupling with the left IFG, we conducted a PPI analysis. Beyond classical GLM analysis, PPI provided insights into the correlation of the time course of distinct brain regions in dependency of the experimental manipulation (Friston et al., 1997). This method detects regions whose activation could be explained by the activation pattern of a seed region in interplay with a specific cognitive or sensory process. In the present study, we aimed to identify the cortical network that is responsible for the processing of sentences comprising of a negation in the main clause, but not to affirmative sentences. The seed region was determined by the main effect of POLARITY_MAIN (negation versus affirmation in the main clause) in the randomeffects analysis. For each participant, the center of the VOI was set to the nearest local maximum to the peak voxel $(-45,29,22)$ in the left IFG. Each VOI had a sphere of $6 \mathrm{~mm}$ radius. The design matrix of each participant comprised of three PPI regressors and the six 
movement parameters (three translation, three rotation). The physiological variable of the PPI was the first regressor. It was the time series of the first eigenvariate of the BOLD signal. The signal was high-pass filtered to account for global signal changes and mean corrected. The psychological variable was the second regressor. It was the experimental context vector convolved with the canonical hemodynamic response function. Trials of conditions with a negation in the main clause were given the value 1 , those conditions with an affirmation in the main clause were given the value -1 , and those of all other conditions 0 . The interaction between the physiological and psychological variable of the PPI was the third regressor. This regressor was created by (1) deconvolving the seed VOI time series, (2) making an element-wise product of it with the psychological variable, (3) convolving with the canonical hemodynamic response function, and (4) orthogonalizing with regards to the other two PPI regressors (Gitelman et al., 2003). The voxels that had a significant context-dependent increase in coupling with the seed region were identified by a $t$-contrast on the third PPI regressor. Individual contrast images entered the second-level random-effect analysis, using one-sample $t$-tests. Two $t$-tests were applied. One testing the contexteffect of negation versus affirmation: Functional coupling with the seed region (left IFG) in the context of negation in the main clause. The other one testing the opposite context-effect, namely affirmation versus negation. Statistical inference was drawn similar to the whole-brain ANOVA (i.e., $p<0.001$, uncorrected).

\section{RESULTS \\ BEHAVIORAL DATA}

An ANOVA on error rates was performed with the factors POLARITY_ MAIN (negation versus affirmation in the main clause) and POLARITY_SUB (negation versus affirmation in the subordinated clause). A significant main effect of POLARITY_MAIN was found $[F(1,16)=10.5, p<0.01]$, indicating that sentences with negations in the main clause induced more errors $(14.4 \%, \mathrm{SD}=11.6)$ than sentences with affirmations in the main clause $(9.7 \%, \mathrm{SD}=10.1$; see Figure 2). The interaction effect of POLARITY_MAIN $\times$ POLARITY_SUB was marginally significant [see Figure $2 ; F(1,16)=4.16, p=0.058$ ] . The step down analysis revealed that the two conditions with negations on the main clause $(\mathrm{NN}=13.1 \%, \mathrm{SD}=11.9 ; \mathrm{NA}=15.8 \%, \mathrm{SD}=11.5)$ produced significantly more errors than the affirmative condition $[\mathrm{AA}=9.4 \%$, $\mathrm{SD}=9.5$; NN-AA: $t(16)=2.25, p<0.05$; NA-AA: $t(16)=4.5, p<0.001]$. The difference between $\mathrm{AN}(10.1 \%, \mathrm{SD}=11.9)$ and NA was also significant $[t(16)=2.75, p<0.05]$. The main effect of POLARITY_SUB did not reach significance $(F<1)$. The analysis of the RTs revealed a main effect of POLARITY_MAIN $[F(1,16)=10.79, p<0.005]$ and an interaction of POLARITY_MAIN $\times$ POLARITY_SUB [see Figure 1; $F(1,16)=70.05, p<0.001]$. The step down analysis revealed that participants responded faster in the AA condition in comparison to the other three conditions $[2.16 \leq t(16) \leq 5.26,0.05 \leq p \leq 0.001]$. RTs of the NA condition were also significantly faster than RTs of the AN condition $[t(16)=2.86, p<0.05]$.

\section{fMRI DATA}

\section{ANOVA}

A whole-brain within-subject ANOVA was performed with the factors POLARITY_MAIN and POLARITY_SUB. The main effect of POLARITY_MAIN revealed activity in left IFG (BA 45), left

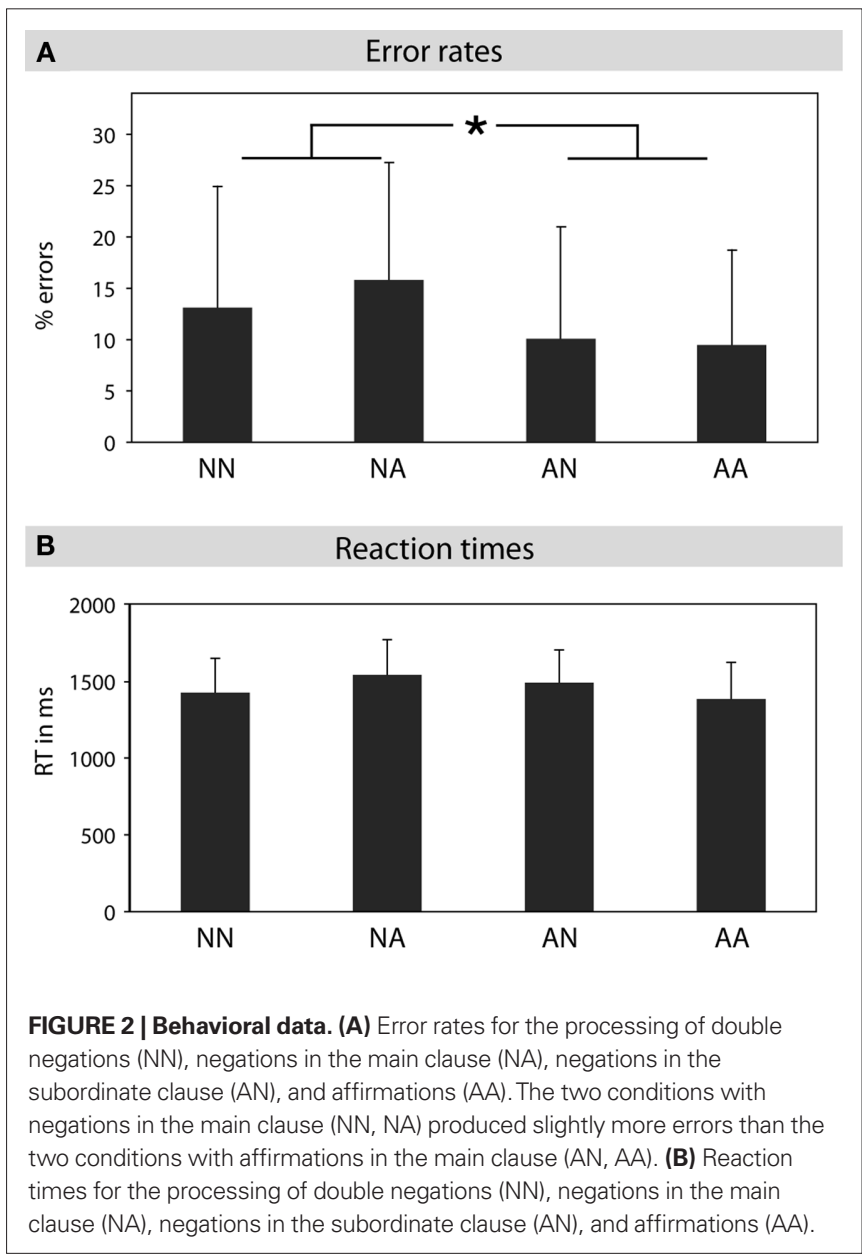

precentral gyrus (BA 6/44), left inferior parietal gyrus (BA 40), right anterior insula (BA 48), and right angular gyrus (BA 39/40; see Table 2 and Figure 3 ). The main effect of POLARITY_SUB and the interaction of POLARITY_MAIN $\times$ POLARITY_SUB did not show any significantly activated voxels.

\section{VOI analysis}

The VOI analysis in left IFG and left IPL corroborated the findings of the whole-brain analysis (see Figure 4). A main effect of POLARITY MAIN was found in the VOI of left IFG $[F(1,15)=13.36, p<0.005]$ and left IPL $[F(1,15)=24.27, p<0.001]$. No other effect was significant $(F s<1.2)$. These results suggest that negative polarity sentences are correlated with higher hemodynamic responses in left IFG and left IPL. The VOI analysis in the right angular gyrus revealed that the activity in this regions was caused by a stronger deactivation of affirmative sentences in comparison to negative sentences. This was true for negations in the main clause [main effect of POLARITY_ MAIN; $F(1,16)=5.87, p<0.05]$ and for negations in the subordinate clause [main effect of POLARITY_SUB; $F(1,16)=11.7, p<0.005$ ]. The interaction of POLARITY_MAIN $\times$ POLARITY_SUB was not significant $(F<0.2)$. The VOI analysis in left precentral gyrus and right anterior insula did not show any significant effects $(F s<1.5)$. Note, that only 13 participants entered that analysis which reduces the statistical power. 


\section{PPI analysis}

The PPI analysis with seed in the left IFG (BA 45) revealed three brain areas, namely left superior temporal gyrus (BA 42), (left) supplementary motor area (BA 6), and left IFG (BA 44/48), see Table 3 and Figure 5 which showed a significant increased functional coupling in the context of negation in the main clause. The opposite contrast-context of affirmation in the main clause - did not show any significantly activated (functionally coupled) brain areas.

\section{DISCUSSION}

The principal finding of the present study is that a network comprising the anterior portion of Broca's area and the left IPL is engaged in the processing of certain types of negations during language comprehension. In particular, the processing of negations in the main clause correlated with activity in BA 45 in the left IFG. The sentences in the study at hand are processed as follows: The polarity of the main clause determines the polarity of the subordinate clause, which is not true the other way round (the polarity of the subordinate clause does not determine the polarity of the main clause). Thus the scope of the negation in the main clause is larger than in the subordinate clause, crossing a clause boundary

Table 2 |Whole-brain ANOVA.

\begin{tabular}{lllllll}
\hline Brain region & BA & Volume & $\boldsymbol{x}$ & $\boldsymbol{y}$ & $\boldsymbol{z}$ & $\boldsymbol{Z}_{\max }$ \\
\hline L IFG (pars triangularis) & 45 & 2079 & -45 & 29 & 22 & 4.39 \\
L precentral G & $44 / 6$ & 513 & -42 & 8 & 37 & 3.61 \\
L inferior parietal/angular G & $39 / 40$ & 7506 & -33 & -55 & 37 & 4.53 \\
R angular/parietal G & $39 / 40$ & 1782 & 39 & -55 & 40 & 3.94 \\
R anterior insula/frontal & 48 & 351 & 36 & 26 & 40 & 3.73 \\
operculum & & & & & &
\end{tabular}

Main effect of Polarity. Anatomical areas, approximate Brodmann's Area (BA), volume (in $\mathrm{mm}^{3}$ ), mean $x, y$, and $z$ Montreal Neurological Institute (MNI) coordinates, and maximal $Z$ values of the significant activations are presented. $L$, left hemisphere; $R$, right hemisphere; $G$, gyrus; IFG, inferior frontal gyrus. in the former but not in the latter case. In the present experiment, we found neuronal correlates for the processing of negations in the main clause. Thus, the activation pattern in the present study is taken to represent higher demands on semantic integration across clauses during processing of sentential negations.

Functional connectivity analyses revealed a functional coupling between Broca's area (BA 45) with the left STG. This coupling depends entirely on the comprehension of negations during sentence processing. In the following these results will be embedded into the framework of semantic processing as well as logical/ conditional reasoning literature. Furthermore, this network will be discussed under neuroanatomical considerations.

\section{NEGATION AS A SEMANTIC FEATURE}

In linguistic terms, the logical form of a sentence is determined by its semantic constituents (e.g., Partee et al., 1993; de Swart, 1998). Moreover, the logical form of a sentence is defined as those syntactic properties that are relevant for semantic interpretations, or in other words: “... the contribution of grammar to meaning.” (May, 1985). The semantic interpretation of the logical form of a sentence can either be affirmative or negative. Thus, polarity is perceived as a semantic property of a sentence. Behavioral studies suggest, that negations are more difficult to integrate into the sentential context than affirmations (e.g., Kaup and Zwaan, 2003; Hasson and Glucksberg, 2006). In the present study the parsing of double negations, single negations, and affirmations were in the scope of investigation. The logical form of sentences (true/false) was systematically varied, while other linguistic features like syntax and phonology were held constant. As a result, the left IFG (pars triangularis, BA 45) was found to be more activated during the processing of negated sentences in comparison to affirmative sentences.

These results are contrary to previous fMRI investigations of sentential negation (Carpenter et al., 1999; Hasegawa et al., 2002; Tettamanti et al., 2008; Christensen, 2009). None of these studies reported increased activity in Broca's area during the processing of negation during sentence comprehension. From the linguistic

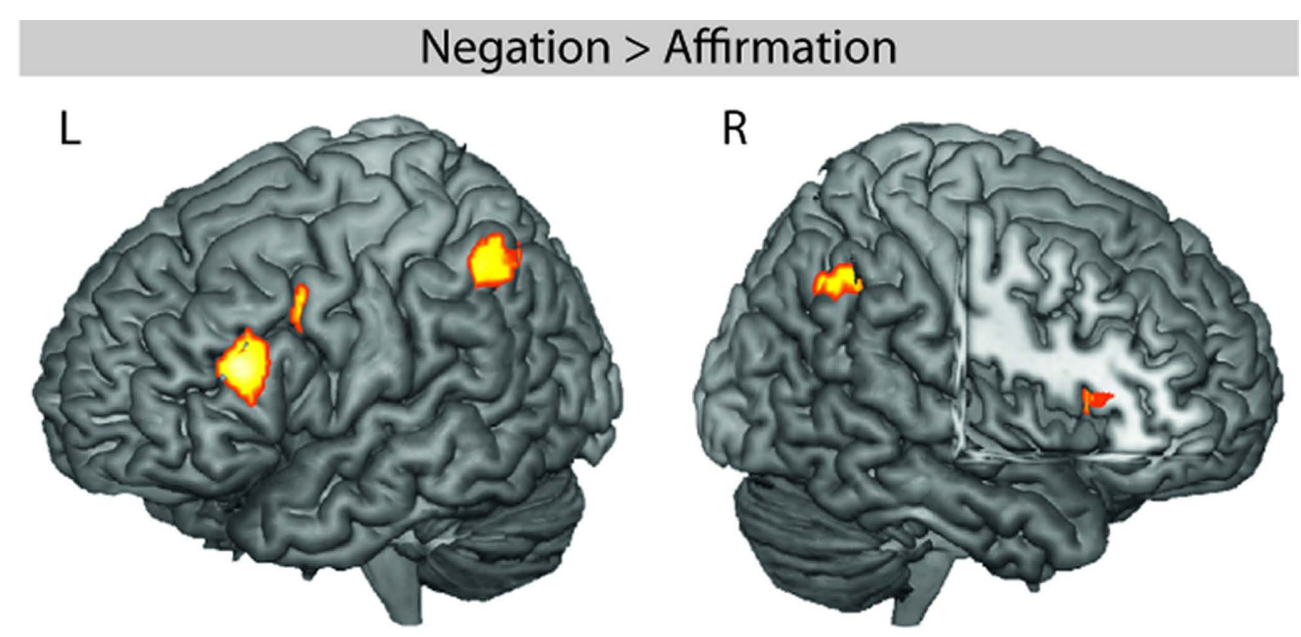

FIGURE 3 | fMRI effect of negation in the main clause. The whole-brain ANOVA revealed significant activity in the left inferior frontal gyrus (LIFG, BA 45), left inferior parietal lobule (LIPL), right angular gyrus (RAG), right anterior insula (RAI), and left precentral gyrus (LPRE). 


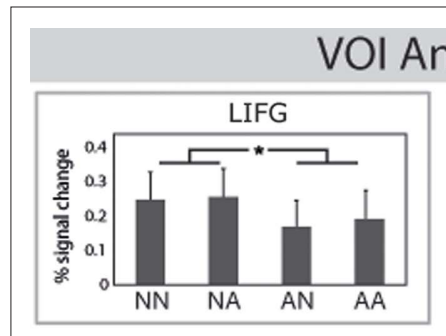

\section{Analysis}
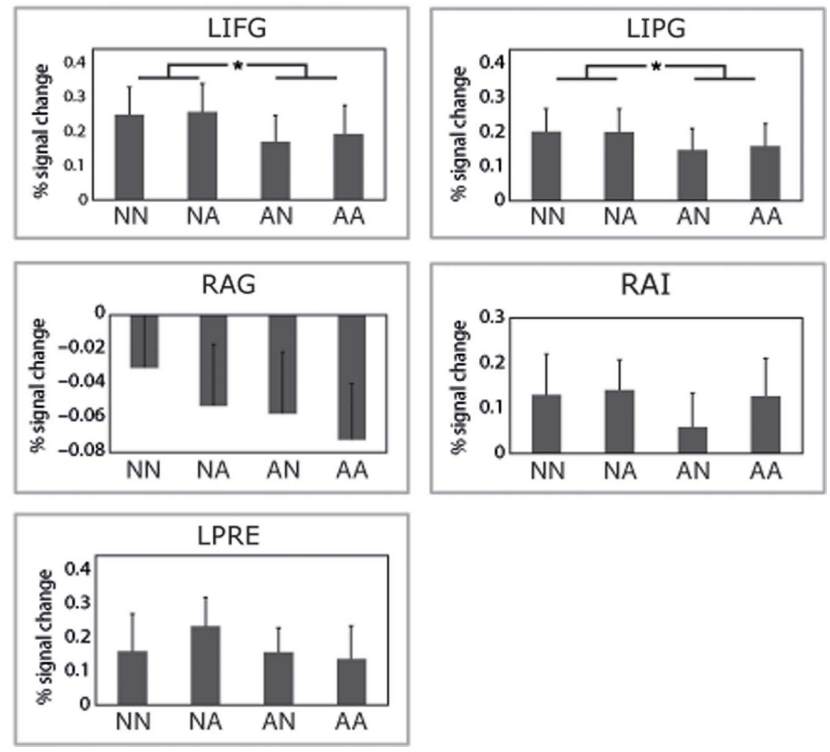

FIGURE 4 | Volume of interest analysis. Top: The VOI analysis in left IFG and left IPG corroborated the findings of the ANOVA. The BOLD response for negations was higher than for affirmations in the main clause. Bottom: The VOI analysis in the right angular gyrus (RAG) revealed more deactivations for affirmations in comparison to negations. The effect in the left precentralis (LPRE) and right anterior insula (RAl) reached not significance.

Table 3 | Psychophysiological interaction analysis.

\begin{tabular}{lrccccc}
\hline Brain region & BA & Volume & $\boldsymbol{x}$ & $\boldsymbol{y}$ & $\boldsymbol{z}$ & $\boldsymbol{Z}_{\max }$ \\
\hline L IFG (pars opercularis) & $44 / 48$ & 270 & -39 & 20 & 19 & 3.81 \\
L superior temporal G & 42 & 324 & -48 & -43 & 19 & 5.52 \\
L supplementary & 6 & 1134 & -15 & -1 & 55 & 3.66 \\
motor area & & & & & & \\
\hline
\end{tabular}

Seed in left IFG (BA 45). Anatomical areas, approximate Brodmann's Area (BA), volume (in $\mathrm{mm}^{3}$ ), mean $x, y$, and $z M N I$ coordinates, and maximal $Z$ values of the brain regions that show significant coupling with the IFG seed region are presented.

L, left hemisphere; G, gyrus; IFG, inferior frontal gyrus.

point of view, a possible explanation of this discrepancy could be that these studies did not investigate the semantic aspect of negation (as the present did). Tettamanti et al. (2008) investigated into the syntactic aspect of negations and Carpenter et al. (1999), Hasegawa et al. (2002), and Christensen (2009) examined pragmatic aspects of negations. Thus, the present findings could suggest, that the processing of negation information relevant for the semantic interpretation of a sentence engages the left IFG. Another possible explanation, however, could be that the study at hand applied not only affirmation and single negations, but also double negations. The studies mentioned above only used affirmations and single negations. Thus, due to the over-learned character of language comprehension processing costs of single negations might be not prominent enough to trigger a significant increase of hemodynamic response in the left IFG.

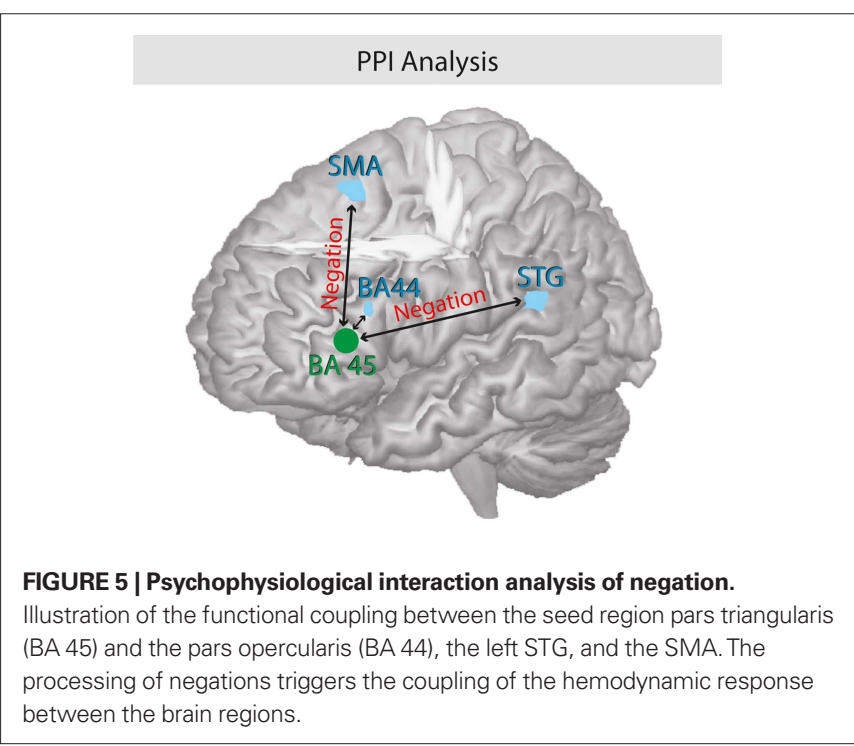

From a broader perspective, a large number of studies have shown that the left IFG is engaged in language processing. In particular, it was proposed that distinctive sub-regions serve different language functions (see Poldrack et al., 1999; Bookheimer, 2002; Vigneau et al., 2006 for recent reviews). Specifically, it was shown that functions like syntax, semantics, and phonology engage different sub-regions of the left IFG. The exact location of the regions involved in the particular language functions differs slightly between the three mentioned reviews. However, all have in common that BA 44 (pars opercularis) is engaged during the processing of syntax, and BA 45/47 (pars triangularis/pars orbitalis) plays a crucial role in semantic processing. The present findings are consistent with these results. The activation in the left pars triangularis as a function of negation in the present study is in line with reported areas for semantic processing in the reviews cited above that analyzed fMRI experiments in the language domain. As far as the present study is concerned, our findings suggest that the left IFG (BA 45) contributes to the processing of negations as a semantic feature.

\section{NEGATION AS AN INSTANCE OF CONDITIONAL REASONING}

Besides the linguistic interpretation of negation as a semantic feature of natural sentences, the present experimental manipulation can also be considered in relation to the mathematical (non-linguistic) concept of conditional reasoning. Experiments exploring conditional reasoning often applied four different types of simple inference problems. The logical problems were created using "if-then" statements like the following: (1) if $p$, then $q=>p$, therefore $q$; (2) if $p$, then not $q=>p$, not $q$; (3) if not $p$, then $q=>$ not $p, q$; (4) if not $p$, then not $q=>p, q$ (e.g., Schoyens et al., 2000). The polarities of these types of inference problems are similar to the four conditions in the present experimental manipulations. We used, however, not conditional "if-then" statements, but "AND" concatenations of statements: $\mathrm{AA}=p$ and $q, \mathrm{AN}=p$ and not $q, \mathrm{NA}=\operatorname{not} p$ and $q, \mathrm{NN}=\operatorname{not} p$ and not $q$. To our knowledge, there is only one functional imaging study using such inference problems as stimuli. Prado and Noveck (2007) reported a para- 
metrical modulation of the hemodynamic response in the right dorsolateral prefrontal cortex (DLPFC) as a function of the four types of inference problems. The response in right DLPFC was highest during the processing of double negations in inference statements. Single negations showed a lower response and affirmations lowest response. There are certain parallels between the study by Prado and Noveck (2007) and the current experiment. It is not only the underlying theoretical principle of conditional reasoning, but also the type of explored independent variables (AA, AN, NA, NN) that are similar. Interestingly, despite of these similarities, the study by Prado and Noveck (2007) and our study found almost completely different brain activation patterns. The only area revealing a higher hemodynamic response as a function of negation in both experiments was the left IPL (BA 40). However, there are also several aspects that differ between the two studies. Prado and Noveck (2007) used conditional reasoning problems ("if-then" statements as mentioned above). In contrast, we used natural sentences comprised of a main and a subordinate clause, representing every day life situations. Taking these two studies into consideration it appears that there is a functional shift between tasks involving "pure" conditional reasoning in logical rules, and tasks involving conditional reasoning embedded in the semantic processing of natural sentences. This functional shift finds it neuronal representation in the prefrontal cortex as a shift between the right DLPFC for conditional reasoning in logical rules (Prado and Noveck, 2007) to the left IFG for conditional reasoning in sentential processing (present study). The left IPL, in contrast, appears to be engaged in processing and resolving of negations, independent of the experimental environment (i.e., logical rules or natural sentences).

From a broader perspective, the (bilateral) IPL has been reported to be implicated in a variety of functions, such as the integration of visuo-spatial processing, cognitive control, and motor operations (see for instance Gottlieb, 2007 for a recent review), working memory (Muller and Knight, 2006), and arithmetic processing (Dehaene et al., 2004). However, recent findings suggest that this region is engaged in semantic processing as well. In a meta-analysis, Binder et al. (2009) identified brain regions to be engaged in semantics, which are not known to be part of the classical perisylvian language network. Using the quantitative approach of activation likelihood estimate (ALE), the authors reported the highest overlap of activation foci between the reviewed experiments in the posterior inferior parietal lobe. This region comprised of the angular gyrus and supramarginal gyrus and corresponds to BA 39/40. The authors concluded that this area seems to play a "role in behaviors requiring fluent conceptual combination, such as sentence comprehension, discourse, problem solving, and planning" (p. 2777). The findings of the present experiment are in line with this interpretation. The comprehension of negations in natural sentences causes more processing costs than the comprehension of affirmative sentences. Moreover, the processing of negations is more demanding in terms of problem solving and planning mechanisms in order to extract the correct content of a sentence. In the present study, the processing of negations during the comprehension of natural sentences generated significantly more activation in the left IPL than the processing of affirmations. The exact location of the activity in this region covered the left inferior parietal (BA 40) and the adjacent angular gyrus (BA 39). Thus, the activation of the left IPL (BA 39/40) as a correlate of negation might reflect higher demands on problem solving and planning mechanisms during sentence comprehension.

\section{FUNCTIONAL COUPLING BETWEEN LEFT IFG, SMA, AND LEFT STG}

In the present study the left anterior IFG and the left IPL were engaged when participants processed negations during sentence comprehension. Moreover, the PPI analysis revealed a functional coupling between left pars triangularis (BA 45) with the adjacent left pars opercularis (BA 44), the SMA (BA 6), and the left STG (BA 42). The coupling of hemodynamic responses in these areas was triggered by the factor of negation in the main clause during online sentence comprehension. Functional imaging studies suggest, that the SMA plays a crucial role in working memory during the rehearsal of phonological information (see Chein et al., 2003 for a review). The functional coupling of left pars triangularis with SMA in the present study might reflect higher demands on verbal working memory during the processing of sentential negations as indicated by the behavioral data.

In contrast, the three perisylvian areas (pars triangularis, pars opercularis, and STG) might have been synchronized due to semantic sentential integration or conditional reasoning processes with negated statements. This finding is consistent with previous functional studies reporting an involvement of the IFG and the posterior superior temporal region for the processing of complex sentences (Bornkessel et al., 2005; Makuuchi et al., 2009). It has been suggested that the left STG could play a crucial role in the integration of syntactic and semantic information during sentence processing as Broca's area and the left STG both have been found to vary as a function of syntactic complexity and difficulty of thematic role assignment (Friederici et al., 2009). In contrast, the processing of syntactic complexity in a hierarchically organized artificial grammar was correlated with activity only in Broca's area (Bahlmann et al., 2008). In line with these results, Tettamanti et al. (2002) and Musso et al. (2003) demonstrated that Broca's area plays a crucial role during the acquisition of new syntactic rules. Bahlmann et al. (2008) applied hierarchically structured syllable sequences as stimuli. Both studies (Bahlmann et al., 2008; Makuuchi et al., 2009) manipulated the hierarchical organization of languagerelated stimuli and found Broca's area to be activated. The left STG, however, comes into play when natural sentences containing semantic information are to be processed, requiring the integration of syntax and semantics (Bornkessel et al., 2005; Makuuchi et al., 2009). The PPI analysis for the present study indicates an increase in functional coupling between left BA 45 and the left STG during the processing of negations. This functional connectivity may be related to neuroanatomical connections in the human brain. The anatomical connection between ventrolateral areas (area 44 and 45) and temporal and parietal regions in the macaque brain was recently investigated by Petrides and Pandya (2009). In this tracer study the authors found a dissociation between a dorsal and a ventral stream connecting ventrolateral areas with temporal and parietal regions. In line with these findings, DTI studies in humans 
suggest dorsal and ventral pathways connecting inferior frontal to temporal and parietal regions (Anwander et al., 2007; Saur et al., 2008; Friederici, 2009). Dorsal pathways connect BA 44 to parietal regions via the superior longitudinal fasciculus and to temporal regions via the branching arcuate fasciculus. Ventral pathways connect BA 45 to the temporal region via the extreme capsule system. The functional coupling between left BA 45 and left STG in the present study might be based on the direct structural connectivity between BA 45 and temporal regions via the extreme capsule. Thus, the present findings suggest, that this connection, plays a crucial role in sentence comprehension, and under the involvement of left BA 44 and SMA, seems to be engaged during the processing of negations.

\section{REFERENCES}

Anwander, A., Tittgemeyer, M., von Cramon, D. Y., Friederici, A. D., and Knosche, T. R. (2007). Connectivitybased parcellation of Broca's area. Cereb. Cortex 17, 816-825.

Bahlmann, J., Schubotz, R. I., and Friederici, A. D. (2008). Hierarchical artificial grammar processing engages Broca's area. Neuroimage 42, 525-534.

Binder, J. R., Desai, R. H., Graves, W. W., and Conant, L. L. (2009). Where is the semantic system? A critical review and meta-analysis of 120 functional neuroimaging studies. Cereb. Cortex 19, 2767-2796.

Bookheimer, S. (2002). Functional MRI of language: new approaches to understanding the cortical organization of semantic processing. Annu. Rev. Neurosci. 25, 151-188.

Bornkessel, I., Zysset, S., Friederici, A. D., von Cramon, D. Y., and Schlesewsky, M. (2005). Who did what to whom? The neural basis of argument hierarchies during language comprehension. Neuroimage 26, 221-233.

Carpenter, P. A., Just, M. A., Keller, T. A., Eddy, W. F., and Thulborn, K. R. (1999). Time course of fMRIactivation in language and spatial networks during sentence comprehension. Neuroimage 10, 216-224.

Chein, J. M., Ravizza, S. M., and Fiez, J. A. (2003). Using neuroimaging to evaluate models of working memory and their implications for language processing. J. Neurolinguistics 16, 315-339.

Christensen, K. R. (2009). Negative and affirmative sentences increase activation in different areas in the brain. $J$. Neurolinguistics 22, 1-17.

Dehaene, S., Molko, N., Cohen, L., and Wilson, A. J. (2004). Arithmetic and the brain. Curr. Opin. Neurobiol. 14, 218-224.

de Swart, H. (1998). Introduction to Natural Language Semantics. Stanford, CA: CSLI Publications.
Fiebach, C. J., Schlesewsky, M., Lohmann, G., von Cramon, D. Y., and Friederici, A. D. (2005). Revisiting the role of Broca's area in sentence processing: syntactic integration versus syntactic working memory. Hum. Brain Mapp. 24, 79-91.

Friederici, A. D. (2009). Pathways to language: fiber tracts in the human brain. Trends Cogn. Sci. 13, 175-181.

Friederici, A. D., Makuuchi, M., and Bahlmann, J. (2009). The role of the posterior superior temporal cortex in sentence comprehension. Neuroreport 20, 563-568.

Friston, K. J., Buechel, C., Fink, G. R., Morris, J., Rolls, E., and Dolan, R. J. (1997). Psychophysiological and modulatory interactions in neuroimaging. Neuroimage 6, 218-229.

Friston, K. J., Holmes, A. P., Worsley, K. J., Poline, J. B., Frith, C. D., and Frackowiak, R. S. (1995). Statistical parametric maps in functional imaging: a general linear approach. Hum. Brain Mapp. 2, 189-210.

Gitelman, D. R., Penny, W. D., Ashburner, J., and Friston, K. J. (2003). Modeling regional and psychophysiologic interactions in $\mathrm{AMRI}$ : the importance of hemodynamic deconvolution. Neuroimage 19, 200-207.

Gottlieb, J. (2007). From thought to action: the parietal cortex as a bridge between perception, action, and cognition. Neuron 53, 9-16.

Griswold, M. A., Jakob, P. M., Heidemann, R. M., Nittka, M., Jellus, V., Wang, J., Kiefer, B., and Haase, A. (2002). Generalized autocalibrating partially parallel acquisitions (GRAPPA). Magn. Reson. Med. 47, 1202-1210.

Hasegawa, M., Carpenter, P. A., and Just, M. A. (2002). An fMRI study of bilingual sentence comprehension and workload. Neuroimage 15, 647-660.

Hasson, U., and Glucksberg, S. (2006). Does understanding negation entail affirmation? An examination of

\section{CONCLUSION}

The present findings demonstrate that the processing of sentential negation engages the left perisylvian language areas as well as the left inferior parietal cortex. It was shown that activity in this network correlates with the variation of polarity information across clause boundaries. The engagement of the left IPL might reflect higher demands on logical or conditional reasoning during the processing of sentential negations. The coupling of the hemodynamic response in the left pars triangularis, left opercularis, and left STG which was stronger during the processing of negated information across clause boundaries than within clause boundaries appears to reflect higher demands on linguistic cross-boundary integration during online comprehension of sentential negation.

negated metaphors. J. Pragmat. 38, 1015-1032.

Just, M. A., and Carpenter, P. A. (1975). The semantics of locative information in pictures and mental images. $\mathrm{Br}$. $\mathrm{J}$. Psychol. 66, 427-441.

Kaup, B., and Zwaan, R. A. (2003). Effects of negation and situational presence on the accessibility of text information. J. Exp. Psychol. Learn. Mem. Cogn. 29, 439-446.

Lohmann, G., Hoehl, S., Brauer J., Danielmeier, C., BornkesselSchlesewsky, I., Bahlmann, J., Turner, R., and Friederici, A. (2010). Setting the frame: the human brain activates a basic low-frequency network for language processing. Cereb. Cortex 20, 1286-1292.

Lüdtke, J., Friedrich, C. K., De Filippis, M., and Kaup, B. (2008). Event-related potential correlates of negation in a sentence-picture verification paradigm. J. Cogn. Neurosci. 20, 1355-1370.

Makuuchi, M., Bahlmann, J., Anwander, A., and Friederici, A. D. (2009). Segregating the core computational faculty of human language from working memory. Proc. Natl. Acad. Sci. U.S.A. 106, 8362-8367.

May, R. (1985). Logical Form: Its Structure and Derivation. Cambridge, MA: MIT Press.

Muller, N. G., and Knight, R. T. (2006). The functional neuroanatomy of working memory: contributions of human brain lesion studies. Neuroscience 139, 51-58.

Musso, M., Moro, A., Glauche, V., Rijntjes, M., Reichenbach, J., Büchel, C., and Weiller, C. (2003). Broca's area and the language instinct. Nat. Neurosci. 6, 774-781.

Partee, B. H., Meulen, A. G. T., and Wall, R. E. (1993). Mathematical Methods in Linguistics (corrected 2nd printing of the 1st Edn.). Dordrecht/Boston/ London: Kluwer Academic Publishers. Petrides, M., and Pandya, D. N. (2009). Distinct parietal and temporal path- ways to the homologues of Broca's area in the monkey. PLoS Biol. 7, e1000170. doi: 10.1371/journal. pbio. 1000170

Poldrack, R. A., Wagner, A. D., Prull, M. W., Desmond, J. E., Glover, G. H., and Gabrieli, J. D. (1999). Functional specialization for semantic and phonological processing in the left inferior prefrontal cortex. Neuroimage 10, 15-35.

Prado, J., and Noveck, I. A. (2007). Overcoming perceptual features in logical reasoning: a parametric functional magnetic resonance imaging study. J. Cogn. Neurosci. 19, 642-657.

Rodd, J. M., Longe, O. A., Randall, B., and Tyler, L. K. (2009). The functional organisation of the fronto-temporal language system: evidence from syntactic and semantic ambiguity. Neuropsychologia 48, 1324-1335.

Saur, D., Kreher, B. W., Schnell, S., Kummerer, D., Kellmeyer, P., Vry, M. S., Umarova, R., Musso, M., Glauche, V., Abel, S., Huber, W., Rijntjes, M., Hennig, J., and Weiller, C. (2008). Ventral and dorsal pathways for language. Proc. Natl.Acad. Sci. U.S.A. 105, 18035-18040.

Schroyens, W., Schaeken, W., Fias, W., and d'Ydewalle, G. (2000). Heuristic and analytic processes in propositional reasoning with negatives. $J$. Exp. Psychol. Learn. Mem. Cogn. 26, 1713-1734.

Tettamanti, M., Alkadhi, H., Moro, A., Perani, D., Kollias, S., and Weniger, D. (2002). Neural correlates for the acquisition of natural language syntax. Neuroimage 17, 700-709.

Tettamanti, M., Manenti, R., Della Rosa, P.A., Falini, A., Perani, D., Cappa, S. F., and Moro, A. (2008). Negation in the brain: modulating action representations. Neuroimage 43, 358-367.

Vigneau, M., Beaucousin, V., Herve, P. Y., Duffau, H., Crivello, F., Houde, O., Mazoyer, B., and Tzourio-Mazoyer, N. (2006). Meta-analyzing left hemi- 
sphere language areas: phonology, semantics, and sentence processing. Neuroimage 30, 1414-1432.

Wartenburger, I., Heekeren, H. R., Abutalebi, J., Cappa, S. F., Villringer, A., and Perani, D. (2003). Early setting of grammatical processing in the bilingual brain. Neuron, 37, 159-170.
Conflict of Interest Statement: The authors declare that the research was conducted in the absence of any commercial or financial relationships that could be construed as a potential conflict of interest.

Received: 21 December 2010; accepted: 10 May 2011; published online: 31 May 2011.
Citation:Bahlmann J, MuellerJL, Makuuch $M$ and Friederici AD (2011) Perisylvian functional connectivity during processing of sentential negation. Front. Psychology 2:104. doi: 10.3389/fpsyg.2011.00104

This article was submitted to Frontiers in Language Sciences, a specialty of Frontiers in Psychology.
Copyright (c) 2011 Bahlmann, Mueller, Makuuchi and Friederici. This is an openaccess article subject to a non-exclusive license between the authors and Frontiers Media $S A$, which permits use, distribution and reproduction in other forums, provided the original authors and source are credited and other Frontiers conditions are complied with. 